\title{
Predicting the Effect of Solvent on the Crystal Habit of Small Organic Molecules Supporting Information
}

\author{
Carl J. Tilbury ${ }^{1}$, Daniel A. Green ${ }^{2}$, William J. Marshall ${ }^{2}$, and Michael F. Doherty ${ }^{1}$ \\ ${ }^{1}$ Department of Chemical Engineering, University of California Santa Barbara, Santa \\ Barbara, United States \\ ${ }^{2}$ DuPont Science \& Technology, Wilmington, Delaware, United States
}

\section{Microkinetic Crystal Growth Model}

The step velocity is calculated using eq 22. Note that the kink density, $\rho_{k, i}$, is a dimensionless probability and the kink rate, $u_{i}$, has units of inverse time. The kink density accounts for the concentration of kink sites along an edge, whereas the kink rate considers the net frequency of attachment at each site and is identical (at steady-state) for each kink along an edge even for noncentrosymmetric cases ${ }^{1}$. The solution-terrace-edge-kink incorporation mechanism will be considered initially. For a centrosymmetric crystal, the kink rate is:

$$
u_{i}=j_{K, i}^{+}-j_{K, i}^{-}
$$

where $j_{K, i}^{+}$and $j_{K, i}^{-}$are the attachment and detachment fluxes to and from a kink on edge $i$, respectively (these rates must also have units of inverse time).

Terrace and edge adsorption can be considered as reversible, elementary reaction processes. The rate of incorporation at a kink on edge $i\left(j_{K, i}^{+}\right.$, eq S.2) should, therefore, be first order in fractional coverage of edge sites with adsorbed growth units $\left(\theta_{E, i}\right.$, which is considered constant for 
a particular edge since edge diffusion is expected to have a lower energetic barrier and be much faster than attachment/detachment events). The attachment rate constant $k_{K, i}^{+}$has units of inverse time (from the frequency prefactor $\nu_{0}$ ) and contains the transition state barrier $\Delta U_{K, i}^{\ddagger}$ that, under solution growth, represents partial desolvation in the kink direction.

$$
j_{K, i}^{+}=k_{K, i}^{+} \theta_{E, i}=\nu_{0} \exp \left(-\frac{\Delta U_{K, i}^{\ddagger}}{k_{B} T}\right) \theta_{E, i}
$$

The reverse (i.e., detachment) rate $\left(j_{K, i}^{-}\right.$, eq S.3) is a zeroth order process with detachment rate constant $k_{K, i}^{-}$(units inverse time) that additionally contains the kink detachment work $\left(\Delta W_{K, i}\right.$, eq 26) as a barrier.

$$
j_{K, i}^{-}=k_{K, i}^{-}=\nu_{0} \exp \left(-\frac{\left(\Delta U_{K, i}^{\ddagger}+\Delta W_{K, i}\right)}{k_{B} T}\right)
$$

To calculate the kink rate, $\theta_{E, i}$ must be determined. To obtain this, one must first consider rates for the preceding processes of terrace and edge adsorption and desorption.

The rate of terrace adsorption $\left(j_{T}^{+}\right.$, eq S.4, units inverse time) can be treated as first order in solute mole fraction in solution $(x)$. Excluding kinks, each terrace site is available for adsorption, so there is no need for a fractional terrace coverage term. The terrace adsorption rate constant $\left(k_{T}^{+}\right.$, units inverse time) contains the transition state desolvation barrier $\Delta U_{T}^{\ddagger}$, which is now in the terrace direction.

$$
j_{T}^{+}=k_{T}^{+} x=\nu_{0} \exp \left(-\frac{\Delta U_{T}^{\ddagger}}{k_{B} T}\right) x
$$

The rate of terrace desorption in the vicinity of edge $i\left(j_{T, i}^{-}\right.$, eq S.5) is instead first order in the fractional coverage of terrace sites around edge $i$ with adsorbed growth units $\left(\theta_{T, i}\right)$. The terrace desorption rate constant $\left(k_{T}^{-}\right.$, units inverse time) additionally contains the terrace detachment work $\left(\Delta W_{T}\right.$, eq 29$)$ as a barrier.

$$
j_{T, i}^{-}=k_{T}^{-} \theta_{T, i}=\nu_{0} \exp \left(-\frac{\left(\Delta U_{T}^{\ddagger}+\Delta W_{T}\right)}{k_{B} T}\right) \theta_{T, i}
$$

The rate of adsorption to edge $i\left(j_{E, i}^{+}\right.$, eq S.6) is first order in $\theta_{T, i}$. Again, excluding kinks, each edge site is available for adsorption, so no fractional edge coverage term is included. The edge adsorption rate constant $\left(k_{E, i}^{+}\right.$, units inverse time) has a transition state desolvation barrier in the 
edge direction, $\Delta U_{E, i}^{\ddagger}$.

$$
j_{E, i}^{+}=k_{E, i}^{+} \theta_{T, i}=\nu_{0} \exp \left(-\frac{\Delta U_{E, i}^{\ddagger}}{k_{B} T}\right) \theta_{T, i}
$$

The rate of desorption from edge $i\left(j_{E, i}^{-}\right.$, eq S.7) is first order in the fractional coverage of edge sites on edge $i$ with adsorbed growth units $\left(\theta_{E, i}\right)$. The edge desorption rate constant $\left(k_{E, i}^{-}\right.$, units inverse time) additionally contains the edge detachment work $\left(\Delta W_{E, i}\right.$, eq 28) as a barrier.

$$
j_{E, i}^{-}=k_{E, i}^{-} \theta_{E, i}=\nu_{0} \exp \left(-\frac{\left(\Delta U_{E, i}^{\ddagger}+\Delta W_{E, i}\right)}{k_{B} T}\right) \theta_{E, i}
$$

The kink rate requires the steady-state fractional edge coverage on edge $i, \theta_{E, i}^{S S}$. Considering how each of the elementary reactions above influence the populations $\theta_{E, i}$ and $\theta_{T, i}$ in the vicinity of a single kink site, the balances shown in eqs S.8 and S.9 result. Note that the solute mole fraction in solution, $x$, is treated as a constant on the timescale of these processes (essentially a large quantity of solution is assumed).

$$
\begin{aligned}
{\left[\frac{d \theta_{E, i}}{d t}=j_{E, i}^{+}+j_{K, i}^{-}-j_{E, i}^{-}-j_{K, i}^{+}\right]_{\theta_{T, i}^{S S}, \theta_{E, i}^{S S}}=0 } \\
{\left[\frac{d \theta_{T, i}}{d t}=j_{T}^{+}+j_{E, i}^{-}-j_{T, i}^{-}-j_{E, i}^{+}\right]_{\theta_{T, i}^{S S}, \theta_{E, i}^{S S}}=0 }
\end{aligned}
$$

Substituting eqs S.2-S.3 and S.6-S.7 into eq S.8 and rearranging, we obtain the steady-state fractional terrace coverage:

$$
\theta_{T, i}^{S S}=\frac{\theta_{E, i}^{S S}\left(k_{E, i}^{-}+k_{K, i}^{+}\right)}{k_{E, i}^{+}}-\frac{k_{K, i}^{-}}{k_{E, i}^{+}}
$$

which can be substituted into eq S.9 together with eqs S.4-S.7, simplifying to:

$$
\theta_{E, i}^{S S}=\frac{k_{E, i}^{+} k_{T}^{+} x+k_{K, i}^{-} k_{T}^{-}+k_{K, i}^{-} k_{E, i}^{+}}{k_{K, i}^{+} k_{E, i}^{+}+k_{K, i}^{+} k_{T}^{-}+k_{E, i}^{-} k_{T}^{-}}
$$

Using eqs S.2, S.3 and S.11 in eq S.1 results in:

$$
u_{i}=\frac{k_{K, i}^{+} k_{E, i}^{+} k_{T}^{+} x-k_{K, i}^{-} k_{E, i}^{-} k_{T}^{-}}{k_{K, i}^{+} k_{E, i}^{+}+k_{K, i}^{+} k_{T}^{-}+k_{E, i}^{-} k_{T}^{-}}
$$

The mole fraction in solution can be written as $x=S x_{\text {sat }}$ (where $S$ is now defined as $S=x / x_{\text {sat }}$ ). 
At $S=1\left(x=x_{s a t}\right)$, the kink rate must be zero to ensure a zero step velocity and no crystal growth or dissolution. Equating $j_{K, i}^{+}=j_{K, i}^{-}$we obtain:

$$
\theta_{E, i}^{s a t}=\frac{k_{K, i}^{-}}{k_{K, i}^{+}}
$$

To ensure a steady-state, $j_{E, i}^{+}=j_{E, i}^{-}$and $j_{T}^{+}=j_{T, i}^{-}$must also be true, which leads to:

$$
\begin{aligned}
\theta_{E, i}^{\text {sat }} & =\frac{k_{E, i}^{+}}{k_{E, i}^{-}} \theta_{T, i}^{\text {sat }} \\
\theta_{T, i}^{\text {sat }} & =\frac{k_{T}^{+}}{k_{T}^{-}} x_{s a t}
\end{aligned}
$$

Using eqs S.13-S.15 we obtain:

$$
x_{s a t}=\frac{k_{K, i}^{-} k_{E, i}^{-} k_{T}^{-}}{k_{K, i}^{+} k_{E, i}^{+} k_{T}^{+}}
$$

Substituting into eq S.12:

$$
u=\sigma \frac{k_{K, i}^{-} k_{E, i}^{-} k_{T}^{-}}{k_{K, i}^{+} k_{E, i}^{+}+k_{K, i}^{+} k_{T}^{-}+k_{E, i}^{-} k_{T}^{-}}
$$

This contains the expected linearity in supersaturation $(\sigma$, which equals $S-1)$ at constant $T$, which has been experimentally measured ${ }^{2-4}$. If $\Delta U_{T}^{\ddagger}+\Delta W_{T}>\Delta U_{E, i}^{\ddagger}$ and $\Delta U_{T}^{\ddagger}+\Delta W_{T}+\Delta W_{E, i}>\Delta U_{K, i}^{\ddagger}$, both of which are expected in the absence of extremely anisotropic interactions, then the first term on the denominator dominates, leading to:

$$
u=\sigma \frac{k_{K, i}^{-} k_{E, i}^{-} k_{T}^{-}}{k_{K, i}^{+} k_{E, i}^{+}}
$$

Upon evaluating rate constants, the kink rate becomes:

$$
u_{i}=\sigma \exp \left(-\frac{\left(\Delta W_{K, i}+\Delta W_{E, i}+\Delta W_{T}\right)}{k_{B} T}\right) \nu_{0} \exp \left(-\frac{\Delta U_{T}^{\ddagger}}{k_{B} T}\right)
$$

which is eq 24 in the text.

Considering the solution-terrace-kink mechanism, one can define rates of kink attachment from/detachment 
to the terrace $\left(j_{K E, i}^{+}\right.$and $j_{K E, i}^{-}$, respectively). The equations become

$$
\begin{aligned}
& u_{i}=j_{K E, i}^{+}-j_{K E, i}^{-} \\
& j_{K E, i}^{+}=k_{K E, i}^{+} \theta_{T, i} \\
& j_{K E, i}^{-}=k_{K E, i}^{-}=k_{K E, i}^{+} \exp \left(-\frac{\left(\Delta W_{K, i}+\Delta W_{E, i}\right)}{k_{B} T}\right) \\
& {\left[\frac{d \theta_{T, i}}{d t}=j_{T}^{+}+j_{K E, i}^{-}-j_{T, i}^{-}-j_{K E, i}^{+}\right]_{\theta_{T, i}^{S S}}=0}
\end{aligned}
$$

Solving for $\theta_{T, i}^{S S}$, substituting into the expression for $u_{i}$ and determining an expression for $x_{\text {sat }}$ reproduces eq S.19 (providing $\Delta U_{T}^{\ddagger}+\Delta W_{T}>\Delta U_{K E, i}^{\ddagger}$ ). Thus, eq S.19 inherently describes both terrace-mediated incorporation mechanisms, so should perform adequately regardless of the kink density.

Note that the extension to non-centrosymmetric molecules must consider $k_{K, i}^{-}, k_{E, i}^{-}$and/or $k_{T}^{-}$ values for each different growth unit present, but the centrosymmetric analysis presented here provides the interesting result that only desolvation in the terrace direction (barrier $\Delta U_{T}^{\ddagger}$ ) is important for the kink rate.

In addition to the kink rate, the $2 \mathrm{D}$ nucleation model (to be detailed in a later publication) requires an expression for $j_{K, i}^{+}$alone; which eq S.24 shows in general form. As described above, the first term in the denominator of eq S.24 is expected to dominate due to the absence of detachment work barriers that exist in the other terms. Similarly, this is also expected for the first term in the numerator (note that in forming eq S.12 the second two terms in the numerator canceled, so such an approximation was unnecessary). Retaining just the dominant first terms and using eq S.16, the resulting expression for $j_{K, i}^{+}$is given in eq S.25.

$$
\begin{aligned}
& j_{K, i}^{+}=k_{K, i}^{+} \theta_{E, i}^{S S}=\frac{k_{K, i}^{+} k_{E, i}^{+} k_{T}^{+} x+k_{K, i}^{+} k_{K, i}^{-} k_{T}^{-}+k_{K, i}^{+} k_{K, i}^{-} k_{E, i}^{+}}{k_{K, i}^{+} k_{E, i}^{+}+k_{K, i}^{+} k_{T}^{-}+k_{E, i}^{-} k_{T}^{-}} \\
& j_{K, i}^{+}=k_{T}^{+} S x_{\text {sat }}=S \exp \left(-\frac{\left(\Delta W_{K, i}+\Delta W_{E, i}+\Delta W_{T}\right)}{k_{B} T}\right) \nu_{0} \exp \left(-\frac{\Delta U_{T}^{\ddagger}}{k_{B} T}\right)
\end{aligned}
$$




\section{Mechanistic Parameters}

Relevant quantities within the mechanistic model (those which enable the crystal habit calculation) are summarized in the sections below for each system studied. For each face family present on a steady-state growth shape prediction, a single face's parameters are provided.

The calculated lattice energy for each crystal can be compared to an experimentally reported sublimation enthalpy. The two quantities differ through a degree of freedom correction, so if the difference between the calculated lattice energy and experimental sublimation enthalpy is of the order RT (which is the case for each system) it lends support to the accuracy of the force field used and, therefore, the energetic calculations within the mechanistic model.

For each face (miller indices $(h k l)), h$ is the step height (a single interplanar spacing, $d_{h k l}$ ) and $R$ is the relative growth rate, where the reference face is indicated on the denominator. If the face is predicted to cross over to $2 \mathrm{D}$ nucleation and growth, the crossover supersaturation $\left(\sigma_{2 D}\right)$ is listed, alongside the supersaturation corresponding to a critical nucleus size of 10 growth units $\left(\sigma_{n_{C}=10}\right)$, which serves as a limit of our modeling applicability within the $2 \mathrm{D}$ regime. Unlike when both faces are in the spiral regime, if a face is growing via $2 \mathrm{D}$ nucleation and growth its relative growth rate is dependent on the supersaturation, so $\sigma$ values for the supplied relative growth rates are indicated where relevant. Further quantities relating to $2 \mathrm{D}$ framework will not be listed here since this portion of the model will be the subject of a future publication, which will provide the appropriate context.

Periodic bond chains (PBCs) on each face are also listed, where their vector [uvw] corresponds to the crystallographic axes. For each PBC, $a_{P}$ is the propagation length, $a_{E}$ is the width of a growth unit along the step, $\alpha_{i, i+1}$ is the angle between that edge and the next and $\phi_{k}$ is the kink energy (note in calculating the kink density, the multi-site expressions developed by Lovette and Doherty $^{5}$ have been used where $\phi_{k} \leq 3 \mathrm{kT}$.

\subsection{Biphenyl}

The lattice energy of biphenyl (CSD code BIPHEN04 ${ }^{6}$ ) is calculated to be $21.3 \mathrm{kcal} / \mathrm{mol}$. The sublimation enthalpy has been reported ${ }^{7}$ as $19.9 \mathrm{kcal} / \mathrm{mol}$ for $T=283-338 \mathrm{~K}$, which corresponds to a difference of $\sim 2$ RT from the lattice energy. 


\subsection{Sublimation Growth}

Table 1 contains the relevant mechanistic parameters for the (001) and (110) faces of biphenyl under sublimation growth. Crystal growth was modeled at $T=313 \mathrm{~K}$, corresponding to the crystals grown by sublimation at $T \sim 40^{\circ} \mathrm{C}$.

Table 1: Biphenyl faces, sublimation growth

\begin{tabular}{llcr}
\hline \multicolumn{4}{l}{ Face (001) } \\
\hline Parameter & & Units & Value \\
\hline & $h$ & $\AA$ & 9.47 \\
& $R=\frac{G_{001}}{G_{001}}$ & - & 1 \\
\hline & $a_{P}$ & $\AA$ & 4.63 \\
{$[1 \overline{1} 0]$ PBC } & $a_{E}$ & $\AA$ & 4.94 \\
& $\alpha_{i, i+1}$ & $\circ$ & 69.5 \\
& $\phi_{k}$ & $\mathrm{kT}$ & 4.39 \\
\hline & $a_{P}$ & $\AA$ & 4.63 \\
{$[110]$ PBC } & $a_{E}$ & $\AA$ & 4.94 \\
& $\alpha_{i, i+1}$ & $\circ$ & 110.5 \\
& $\phi_{k}$ & $\mathrm{kT}$ & 4.39 \\
\hline
\end{tabular}

\begin{tabular}{clcr}
\hline \multicolumn{3}{l}{ Face (110) } & \\
\hline Parameter & & Units & Value \\
\hline & $h$ & $\AA$ & 4.62 \\
& $R=\frac{G_{110}}{G_{001}}$ & - & 2.43 \\
\hline & $a_{P}$ & $\AA$ & 9.48 \\
{$[1 \overline{1} 0]$ PBC } & $a_{E}$ & $\AA$ & 4.94 \\
& $\alpha_{i, i+1}$ & $\circ$ & 85.8 \\
& $\phi_{k}$ & $\mathrm{kT}$ & 4.39 \\
\hline & $a_{P}$ & $\AA$ & 4.93 \\
{$[001] \mathrm{PBC}$} & $a_{E}$ & $\AA$ & 9.51 \\
& $\alpha_{i, i+1}$ & $\circ$ & 94.2 \\
& $\phi_{k}$ & $\mathrm{kT}$ & 1.35 \\
\hline
\end{tabular}

\subsection{Growth from Toluene}

Table 2 contains the relevant mechanistic parameters for the (001) and (110) faces of biphenyl grown from toluene. Crystal growth was modeled at $T=302 \mathrm{~K}$, corresponding to the experimentally reported ${ }^{8}$ crystals grown from toluene at $T=28.85^{\circ} \mathrm{C}$. 
Table 2: Biphenyl faces, growth from toluene

\begin{tabular}{|c|c|c|c|}
\hline \multicolumn{4}{|c|}{ Face (001) } \\
\hline \multirow[t]{3}{*}{ Parameter } & & Units & Value \\
\hline & $h$ & $\AA$ & 9.47 \\
\hline & $R=\frac{G_{001}}{G_{001}}$ & - & 1 \\
\hline \multirow{4}{*}{ [010] PBC } & $a_{P}$ & $\AA$ & 4.06 \\
\hline & $a_{E}$ & $\AA$ & 5.63 \\
\hline & $\alpha_{i, i+1}$ & 0 & 55.3 \\
\hline & $\phi_{k}$ & $\mathrm{kT}$ & 0.065 \\
\hline \multirow{4}{*}[1\overline{1}0]{$\mathrm{PBC}$} & $a_{P}$ & $\AA$ & 4.63 \\
\hline & $a_{E}$ & $\AA$ & 4.94 \\
\hline & $\alpha_{i, i+1}$ & $\circ$ & 69.5 \\
\hline & $\phi_{k}$ & $\mathrm{kT}$ & 0.204 \\
\hline \multirow{4}{*}{ [110] PBC } & $a_{P}$ & $\AA$ & 4.63 \\
\hline & $a_{E}$ & $\AA$ & 4.94 \\
\hline & $\alpha_{i, i+1}$ & $\circ$ & 55.3 \\
\hline & $\phi_{k}$ & $\mathrm{kT}$ & 0.204 \\
\hline
\end{tabular}

\begin{tabular}{|c|c|c|c|}
\hline \multicolumn{4}{|c|}{ Face (110) } \\
\hline Parameter & & Units & Value \\
\hline \multirow{5}{*}{$\begin{array}{l}(\sigma=0.006) \\
(\sigma=0.007)\end{array}$} & $h$ & $\AA$ & 4.62 \\
\hline & $R=\frac{G_{110}}{G_{001}}$ & - & 3.2 \\
\hline & $R=\frac{G_{110}}{G_{001}}$ & - & 15 \\
\hline & $\sigma_{2 D}$ & - & 0.005 \\
\hline & $\sigma_{n_{C}=10}$ & - & 0.1 \\
\hline \multirow{4}{*}{ [001] PBC } & $a_{P}$ & $\AA$ & 4.93 \\
\hline & $a_{E}$ & $\AA$ & 9.51 \\
\hline & $\alpha_{i, i+1}$ & 0 & 28.3 \\
\hline & $\phi_{k}$ & kT & 0.021 \\
\hline \multirow{4}{*}[\overline{1}1\overline{2}]{$\mathrm{PBC}$} & $a_{P}$ & $\AA$ & 4.51 \\
\hline & $a_{E}$ & $\AA$ & 10.4 \\
\hline & $\alpha_{i, i+1}$ & o & 65.9 \\
\hline & $\phi_{k}$ & $\mathrm{kT}$ & 0.093 \\
\hline \multirow{4}{*}[1\overline{1}0]{$\mathrm{PBC}$} & $a_{P}$ & $\AA$ & 9.48 \\
\hline & $a_{E}$ & $\AA$ & 4.94 \\
\hline & $\alpha_{i, i+1}$ & 0 & 85.8 \\
\hline & $\phi_{k}$ & kT & 0.204 \\
\hline
\end{tabular}

\subsection{Adipic Acid}

The lattice energy of adipic acid (CSD code $\mathrm{ADIPAC}^{9}$ ) is calculated to be $31.9 \mathrm{kcal} / \mathrm{mol}$. The sublimation enthalpy has been reported ${ }^{10}$ as $30.8 \mathrm{kcal} / \mathrm{mol}$ for $T=298-353 \mathrm{~K}$, which corresponds to a difference of $\sim 2$ RT from the lattice energy.

\subsection{Sublimation Growth}

Table 3 contains the relevant mechanistic parameters for the (011) and (100) faces of adipic acid under sublimation growth. Crystal growth was modeled at $T=433 \mathrm{~K}$, corresponding to the crystals grown by sublimation at $T \sim 160^{\circ} \mathrm{C}$.

\subsection{Growth from Water}

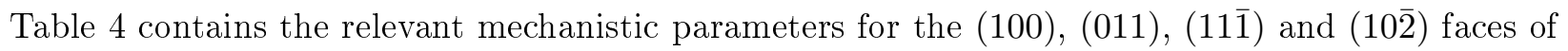
adipic acid when grown from water. Crystal growth was modeled at $T=307 \mathrm{~K}$, corresponding to 
Table 3: Adipic acid faces, sublimation growth

\begin{tabular}{|c|c|c|c|}
\hline \multicolumn{4}{|c|}{ Face (011) } \\
\hline \multirow[t]{3}{*}{ Parameter } & & Units & Value \\
\hline & $h$ & $\AA$ & 4.13 \\
\hline & $R=\frac{G_{011}}{G_{011}}$ & - & 1 \\
\hline \multirow{4}{*}{ [100] PBC } & $a_{P}$ & $\bar{\AA}$ & 4.30 \\
\hline & $a_{E}$ & $\AA$ & 10.0 \\
\hline & $\alpha_{i, i+1}$ & $\circ$ & 49.6 \\
\hline & $\phi_{k}$ & $\mathrm{kT}$ & 5.53 \\
\hline \multirow{4}{*}{ [0̄11] PBC } & $a_{P}$ & $\AA$ & 7.62 \\
\hline & $a_{E}$ & $\AA$ & 5.65 \\
\hline & $\alpha_{i, i+1}$ & $\circ$ & 130.4 \\
\hline & $\phi_{k}$ & $\mathrm{kT}$ & 2.60 \\
\hline
\end{tabular}

\begin{tabular}{clcr}
\hline \multicolumn{3}{l}{ Face (100) } & \\
\hline Parameter & & Units & Value \\
\hline & $h$ & $\AA$ & 6.86 \\
& $R=\frac{G_{100}}{G_{011}}$ & - & 14.0 \\
\hline & $a_{P}$ & $\AA$ & 4.58 \\
{$[0 \overline{1} \overline{1}]$ PBC } & $a_{E}$ & $\AA$ & 5.65 \\
& $\alpha_{i, i+1}$ & $\circ$ & 54.2 \\
& $\phi_{k}$ & $\mathrm{kT}$ & 2.60 \\
\hline & $a_{P}$ & $\AA$ & 4.58 \\
{$[0 \overline{1} 1] \mathrm{PBC}$} & $a_{E}$ & $\AA$ & 5.65 \\
& $\alpha_{i, i+1}$ & $\circ$ & 125.8 \\
& $\phi_{k}$ & $\mathrm{kT}$ & 2.60 \\
\hline
\end{tabular}

experimentally reported ${ }^{11}$ crystals grown from water at $T=34^{\circ} \mathrm{C}$. 
Table 4: Adipic acid faces, grown from water

\begin{tabular}{|c|c|c|c|}
\hline \multicolumn{4}{|c|}{ Face (100) } \\
\hline \multirow[t]{3}{*}{ Parameter } & & Units & Value \\
\hline & $h$ & $\AA$ & 6.86 \\
\hline & $R=\frac{G_{100}}{G_{100}}$ & - & 1 \\
\hline \multirow{4}{*}{ [010] PBC } & $a_{P}$ & $\AA$ & 5.03 \\
\hline & $a_{E}$ & $\AA$ & 5.15 \\
\hline & $\alpha_{i, i+1}$ & $\circ$ & 62.9 \\
\hline & $\phi_{k}$ & $\mathrm{kT}$ & 5.19 \\
\hline \multirow{4}{*}[0\overline{1}\overline{1}]{$\mathrm{PBC}$} & $a_{P}$ & $\AA$ & 4.58 \\
\hline & $a_{E}$ & $\AA$ & 5.65 \\
\hline & $\alpha_{i, i+1}$ & $\circ$ & 54.2 \\
\hline & $\phi_{k}$ & $\mathrm{kT}$ & 5.63 \\
\hline \multirow{4}{*}{ [01̄1] PBC } & $a_{P}$ & $\AA$ & 4.58 \\
\hline & $a_{E}$ & $\AA$ & 5.65 \\
\hline & $\alpha_{i, i+1}$ & $\circ$ & 62.9 \\
\hline & $\phi_{k}$ & $\mathrm{kT}$ & 5.63 \\
\hline \multicolumn{4}{|c|}{ Face $(11 \overline{1})$} \\
\hline \multirow[t]{3}{*}{ Parameter } & & Units & Value \\
\hline & $h$ & $\AA$ & 4.51 \\
\hline & $R=\frac{G_{11 \overline{1}}}{G_{100}}$ & - & 1.89 \\
\hline \multirow{4}{*}{ 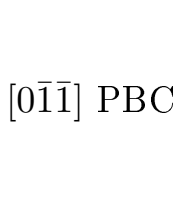 } & $a_{P}$ & $\AA$ & 6.97 \\
\hline & $a_{E}$ & $\AA$ & 5.65 \\
\hline & $\alpha_{i, i+1}$ & $\circ$ & 114.6 \\
\hline & $\phi_{k}$ & $\mathrm{kT}$ & 5.63 \\
\hline \multirow{4}{*}[2\overline{1}1]{$\mathrm{PBC}$} & $a_{P}$ & $\bar{\AA}$ & $\overline{5.14}$ \\
\hline & $a_{E}$ & $\AA$ & 7.67 \\
\hline & $\alpha_{i, i+1}$ & $\circ$ & 65.4 \\
\hline & $\phi_{k}$ & $\mathrm{kT}$ & 3.86 \\
\hline
\end{tabular}

\begin{tabular}{|c|c|c|c|}
\hline \multicolumn{4}{|c|}{ Face $(10 \overline{2})$} \\
\hline \multirow[t]{3}{*}{ Parameter } & & Units & Value \\
\hline & $h$ & $\bar{\AA}$ & 4.78 \\
\hline & $R=\frac{G_{10 \overline{2}}}{G_{100}}$ & - & 2.46 \\
\hline \multirow{4}{*}{ [010] PBC } & $a_{P}$ & $\AA$ & 7.22 \\
\hline & $a_{E}$ & $\AA$ & 5.15 \\
\hline & $\alpha_{i, i+1}$ & $\circ$ & 70.4 \\
\hline & $\phi_{k}$ & $\mathrm{kT}$ & 5.19 \\
\hline \multirow{4}{*}[\overline{2}\overline{1}\overline{1}]{$\mathrm{PBC}$} & $a_{P}$ & $\bar{\AA}$ & 4.85 \\
\hline & $a_{E}$ & $\AA$ & 7.67 \\
\hline & $\alpha_{i, i+1}$ & $\circ$ & 39.2 \\
\hline & $\phi_{k}$ & $\mathrm{kT}$ & 3.86 \\
\hline \multirow{4}{*}{ [2̄11] PBC } & $a_{P}$ & $\bar{\AA}$ & 4.85 \\
\hline & $a_{E}$ & $\AA$ & 7.67 \\
\hline & $\alpha_{i, i+1}$ & $\circ$ & 70.4 \\
\hline & $\phi_{k}$ & $\mathrm{kT}$ & 3.86 \\
\hline \multicolumn{4}{|c|}{ Face (011) } \\
\hline \multirow[t]{3}{*}{ Parameter } & & Units & Value \\
\hline & $h$ & $\AA$ & 4.13 \\
\hline & $R=\frac{G_{011}}{G_{100}}$ & - & 1.79 \\
\hline \multirow{4}{*}{ [0̄1] PBC } & $a_{P}$ & $\AA$ & 7.62 \\
\hline & $a_{E}$ & $\AA$ & 5.65 \\
\hline & $\alpha_{i, i+1}$ & $\circ$ & 96.3 \\
\hline & $\phi_{k}$ & $\mathrm{kT}$ & 5.63 \\
\hline \multirow{4}{*}{ 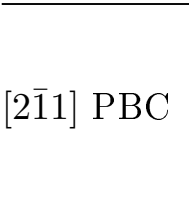 } & $a_{P}$ & $\AA$ & 5.62 \\
\hline & $a_{E}$ & $\AA$ & 7.67 \\
\hline & $\alpha_{i, i+1}$ & $\circ$ & 83.7 \\
\hline & $\phi_{k}$ & $\mathrm{kT}$ & 3.86 \\
\hline
\end{tabular}




\subsection{Pentaerythritol}

The lattice energy of pentaerythritol (CSD code PERYTO04 ${ }^{12}$ ) is calculated to be $44.1 \mathrm{kcal} / \mathrm{mol}$. The sublimation enthalpy has been reported $^{13}$ as $38.5 \mathrm{kcal} / \mathrm{mol}$ for $T=418-455 \mathrm{~K}$, which corresponds to a difference of $\sim 6$ RT from the lattice energy.

\subsection{Sublimation Growth}

Table 5 contains the relevant mechanistic parameters for the $(002),(101)$ and $(10 \overline{1})$ faces of pentaerythritol under sublimation growth. Crystal growth was modeled at $T=413 \mathrm{~K}$, corresponding to the crystals grown by sublimation at $T \sim 140^{\circ} \mathrm{C}$.

\subsection{Growth from Water}

Table 6 contains the relevant mechanistic parameters for the (101), (101) and (110) faces of pentaerythritol when grown from water. Crystal growth was modeled at $T=313 \mathrm{~K}$ (within the range of typical experimentally reported ${ }^{14,15}$ temperatures for pentaerythritol grown from water). 
Table 5: Pentaerythritol faces, sublimation growth

\begin{tabular}{clcr}
\hline \multicolumn{3}{l}{ Face (002) } \\
\hline Parameter & & Units & Value \\
\hline & $h$ & $\AA$ & 4.38 \\
& $R=\frac{G_{002}}{G_{002}}$ & - & 1 \\
\hline & $a_{P}$ & $\AA$ & 6.09 \\
{$[100] \mathrm{PBC}$} & $a_{E}$ & $\AA$ & 6.09 \\
& $\alpha_{i, i+1}$ & $\circ$ & 90 \\
& $\phi_{k}$ & $\mathrm{kT}$ & 7.85 \\
\hline \multirow{5}{*}[010]{$\mathrm{PBC}$} & $a_{P}$ & $\AA$ & 6.09 \\
& $a_{i, i+1}$ & $\circ$ & 6.09 \\
& $\phi_{k}$ & $\mathrm{kT}$ & 9.85 \\
\hline
\end{tabular}

\begin{tabular}{clcc}
\hline \multicolumn{4}{l}{ Face (101) } \\
\hline Parameter & & Units & Value \\
\hline & $h$ & $\AA$ & 5.00 \\
& $R=\frac{G_{101}}{G_{002}}$ & - & 9.34 \\
\hline & $a_{P}$ & $\AA$ & 5.33 \\
{$[0 \overline{1} 0]$ PBC } & $a_{E}$ & $\AA$ & 6.09 \\
& $\alpha_{i, i+1}$ & $\circ$ & 60.3 \\
& $\phi_{k}$ & $\mathrm{kT}$ & 7.85 \\
\hline & $a_{P}$ & $\AA$ & 5.29 \\
{$[1 \overline{1} \overline{1}]$ PBC } & $a_{E}$ & $\AA$ & 6.14 \\
& $\alpha_{i, i+1}$ & $\circ$ & 59.4 \\
& $\phi_{k}$ & $\mathrm{kT}$ & 1.88 \\
\hline & $a_{P}$ & $\AA$ & 5.29 \\
{$[\overline{1} \overline{1} 1]$ PBC } & $a_{E}$ & $\AA$ & 6.14 \\
& $\alpha_{i, i+1}$ & $\circ$ & 60.3 \\
& $\phi_{k}$ & $\mathrm{kT}$ & 1.88 \\
\hline
\end{tabular}

\begin{tabular}{|c|c|c|c|}
\hline \multicolumn{4}{|c|}{ Face $(10 \overline{1})$} \\
\hline \multirow[t]{3}{*}{ Parameter } & & Units & Value \\
\hline & $h$ & $\AA$ & 5.00 \\
\hline & $R=\frac{G_{10 \overline{1}}}{G_{002}}$ & - & 9.34 \\
\hline \multirow{4}{*}[0\overline{1}0]{$\mathrm{PBC}$} & $a_{P}$ & $\AA$ & 5.33 \\
\hline & $a_{E}$ & $\AA$ & 6.09 \\
\hline & $\alpha_{i, i+1}$ & $\circ$ & 60.3 \\
\hline & $\phi_{k}$ & $\mathrm{kT}$ & 7.85 \\
\hline \multirow{4}{*}{ [111] PBC } & $a_{P}$ & $\AA$ & 5.29 \\
\hline & $a_{E}$ & $\AA$ & 6.14 \\
\hline & $\alpha_{i, i+1}$ & $\circ$ & 59.4 \\
\hline & $\phi_{k}$ & $\mathrm{kT}$ & 1.88 \\
\hline \multirow{4}{*}{ [1]11] PBC } & $a_{P}$ & $\AA$ & 5.29 \\
\hline & $a_{E}$ & $\AA$ & 6.14 \\
\hline & $\alpha_{i, i+1}$ & $\circ$ & 60.3 \\
\hline & $\phi_{k}$ & $\mathrm{kT}$ & 1.88 \\
\hline
\end{tabular}


Table 6: Pentaerythritol faces, growth from water

\begin{tabular}{llcr}
\hline \multicolumn{4}{l}{ Face (101) } \\
\hline Parameter & & Units & Value \\
\hline & $h$ & $\AA$ & 5.00 \\
& $R=\frac{G_{101}}{G_{101}}$ & - & 1 \\
\hline & $a_{P}$ & $\AA$ & 5.29 \\
{$[1 \overline{1} \overline{1}]$ PBC } & $a_{E}$ & $\AA$ & 6.14 \\
& $\alpha_{i, i+1}$ & $\circ$ & 59.4 \\
& $\phi_{k}$ & $\mathrm{kT}$ & 4.36 \\
\hline & $a_{P}$ & $\AA$ & 5.29 \\
{$[\overline{1} \overline{1} 1] \mathrm{PBC}$} & $a_{E}$ & $\AA$ & 6.14 \\
& $\alpha_{i, i+1}$ & $\circ$ & 120.6 \\
& $\phi_{k}$ & $\mathrm{kT}$ & 4.36 \\
\hline
\end{tabular}

\begin{tabular}{clcr}
\hline \multicolumn{4}{l}{ Face $(10 \overline{1})$} \\
\hline Parameter & & Units & Value \\
\hline & $h$ & $\AA$ & 5.00 \\
& $R=\frac{G_{10 \overline{1}}}{G_{101}}$ & - & 1 \\
\hline & $a_{P}$ & $\AA$ & 5.29 \\
{$[111]$ PBC } & $a_{E}$ & $\AA$ & 6.14 \\
& $\alpha_{i, i+1}$ & $\circ$ & 59.4 \\
& $\phi_{k}$ & $\mathrm{kT}$ & 4.36 \\
\hline & $a_{P}$ & $\AA$ & 5.29 \\
{$[1 \overline{1} 1] \mathrm{PBC}$} & $a_{E}$ & $\AA$ & 6.14 \\
& $\alpha_{i, i+1}$ & $\circ$ & 120.6 \\
& $\phi_{k}$ & $\mathrm{kT}$ & 4.36 \\
\hline
\end{tabular}

\begin{tabular}{llcr}
\hline \multicolumn{4}{l}{ Face $(110)$} \\
\hline Parameter & & Units & Value \\
\hline & $h$ & $\AA$ & 4.30 \\
$(\sigma<0.15)$ & $R=\frac{G_{110}}{G_{101}}$ & - & 0.86 \\
$(\sigma=0.18)$ & $R=\frac{G_{110}}{G_{101}}$ & - & 1.87 \\
& $\sigma_{2 D}$ & - & 0.15 \\
& $\sigma_{n_{C}=10}$ & - & 0.61 \\
\hline & $a_{P}$ & $\AA$ & 6.14 \\
{$[1 \overline{1} \overline{1}] \mathrm{PBC}$} & $a_{E}$ & $\AA$ & 6.14 \\
& $\alpha_{i, i+1}$ & $\circ$ & 89.0 \\
& $\phi_{k}$ & $\mathrm{kT}$ & 4.36 \\
\hline & $a_{P}$ & $\AA$ & 6.14 \\
{$[1 \overline{1} 1] \mathrm{PBC}$} & $a_{E}$ & $\AA$ & 6.14 \\
& $\alpha_{i, i+1}$ & $\circ$ & 91.0 \\
& $\phi_{k}$ & $\mathrm{kT}$ & 4.36 \\
\hline & & &
\end{tabular}




\subsection{Naphthalene}

The lattice energy of naphthalene (CSD code NAPHTA10 ${ }^{16}$ ) is calculated to be $18.5 \mathrm{kcal} / \mathrm{mol}$. The sublimation enthalpy has been reported ${ }^{17}$ as $18.2 \mathrm{kcal} / \mathrm{mol}$ for $T=328-398 \mathrm{~K}$, which corresponds to a difference of $\sim 0.5 \mathrm{RT}$ from the lattice energy.

\subsection{Sublimation Growth}

Table 7 contains the relevant mechanistic parameters for the $(001),(11 \overline{1}),(110)$ and $(20 \overline{1})$ faces of naphthalene under sublimation growth. Crystal growth was modeled at $T=283 \mathrm{~K}$, corresponding to the growth temperature of experimentally reported ${ }^{18}$ sublimation-grown crystals.

\subsection{Growth from Ethanol}

Table 8 contains the relevant mechanistic parameters for the $(001),(11 \overline{1}),(110)$ and $(20 \overline{1})$ faces of naphthalene when grown from ethanol. Crystal growth was modeled at $T=291 \mathrm{~K}$, corresponding to the experimentally reported ${ }^{18}$ crystals grown from ethanol.

\subsection{Growth from Cyclohexane}

Table 9 contains the relevant mechanistic parameters for the (001), (110) and (201) faces of naphthalene when grown from cyclohexane. Crystal growth was modeled at $T=290 \mathrm{~K}$, corresponding to the experimentally reported ${ }^{18}$ crystals grown from cyclohexane. 
Table 7: Naphthalene faces, sublimation growth

\begin{tabular}{|c|c|c|c|}
\hline \multicolumn{4}{|c|}{ Face (001) } \\
\hline \multirow[t]{3}{*}{ Parameter } & & Units & $\overline{\text { Value }}$ \\
\hline & $h$ & $\AA$ & 7.24 \\
\hline & $R=\frac{G_{001}}{G_{001}}$ & - & 1 \\
\hline \multirow{4}{*}{ [110] PBC } & $a_{P}$ & $\AA$ & 4.83 \\
\hline & $a_{E}$ & $\AA$ & 5.08 \\
\hline & $\alpha_{i, i+1}$ & $\circ$ & 72.1 \\
\hline & $\phi_{k}$ & $\mathrm{kT}$ & 3.85 \\
\hline \multirow{4}{*}{ [110] PBC } & $a_{P}$ & $\AA$ & 4.83 \\
\hline & $a_{E}$ & $\AA$ & 5.08 \\
\hline & $\alpha_{i, i+1}$ & $\circ$ & 107.9 \\
\hline & $\phi_{k}$ & $\mathrm{kT}$ & 3.85 \\
\hline \multicolumn{4}{|c|}{ Face (110) } \\
\hline \multirow[t]{3}{*}{ Parameter } & & Units & Value \\
\hline & $h$ & $\AA$ & $\overline{4.50}$ \\
\hline & $R=\frac{G_{110}}{G_{001}}$ & - & 2.89 \\
\hline \multirow{4}{*}[\overline{1}1\overline{2}]{$\mathrm{PBC}$} & $a_{P}$ & $\AA$ & 5.02 \\
\hline & $a_{E}$ & $\AA$ & 7.86 \\
\hline & $\alpha_{i, i+1}$ & $\circ$ & 81.1 \\
\hline & $\phi_{k}$ & $\mathrm{kT}$ & 1.27 \\
\hline \multirow{4}{*}{ [110] PBC } & $a_{P}$ & $\AA$ & 7.77 \\
\hline & $a_{E}$ & $\AA$ & 5.08 \\
\hline & $\alpha_{i, i+1}$ & $\circ$ & 98.9 \\
\hline & $\phi_{k}$ & $\mathrm{kT}$ & 3.85 \\
\hline
\end{tabular}

\begin{tabular}{|c|c|c|c|}
\hline \multicolumn{4}{|c|}{ Face $(11 \overline{1})$} \\
\hline \multirow[t]{3}{*}{ Parameter } & & Units & Value \\
\hline & $h$ & $\AA$ & 4.65 \\
\hline & $R=\frac{G_{11 \overline{1}}}{G_{001}}$ & - & 2.98 \\
\hline \multirow{4}{*}{ [110] PBC } & $a_{P}$ & $\AA$ & 7.52 \\
\hline & $a_{E}$ & $\AA$ & 5.08 \\
\hline & $\alpha_{i, i+1}$ & $\circ$ & 73.0 \\
\hline & $\phi_{k}$ & $\mathrm{kT}$ & 3.85 \\
\hline \multirow{4}{*}[\overline{1}\overline{1}\overline{2}]{$\mathrm{PBC}$} & $a_{P}$ & $\AA$ & 4.86 \\
\hline & $a_{E}$ & $\AA$ & 7.86 \\
\hline & $\alpha_{i, i+1}$ & $\circ$ & 107.0 \\
\hline & $\phi_{k}$ & $\mathrm{kT}$ & 1.27 \\
\hline \multicolumn{4}{|c|}{ Face $(20 \overline{1})$} \\
\hline \multirow[t]{3}{*}{ Parameter } & & Units & Value \\
\hline & $h$ & $\bar{\AA}$ & 4.09 \\
\hline & $R=\frac{G_{20 \overline{1}}}{G_{001}}$ & - & 3.84 \\
\hline \multirow{4}{*}{ [010] PBC } & $a_{P}$ & $\AA$ & 7.27 \\
\hline & $a_{E}$ & $\AA$ & 5.97 \\
\hline & $\alpha_{i, i+1}$ & $\circ$ & 67.7 \\
\hline & $\phi_{k}$ & $\mathrm{kT}$ & 2.99 \\
\hline \multirow{4}{*}[\overline{1}\overline{1}\overline{2}]{$\mathrm{PBC}$} & $a_{P}$ & $\AA$ & 5.53 \\
\hline & $a_{E}$ & $\AA$ & 7.86 \\
\hline & $\alpha_{i, i+1}$ & $\circ$ & 44.6 \\
\hline & $\phi_{k}$ & $\mathrm{kT}$ & 1.27 \\
\hline \multirow{4}{*}[\overline{1}1\overline{2}]{$\mathrm{PBC}$} & $a_{P}$ & $\AA$ & 5.53 \\
\hline & $a_{E}$ & $\AA$ & 7.86 \\
\hline & $\alpha_{i, i+1}$ & $\circ$ & 67.7 \\
\hline & $\phi_{k}$ & kT & 1.27 \\
\hline
\end{tabular}


Table 8: Naphthalene faces, grown from ethanol

\begin{tabular}{|c|c|c|c|}
\hline \multicolumn{4}{|c|}{ Face (001) } \\
\hline \multirow[t]{3}{*}{ Parameter } & & Units & Value \\
\hline & $h$ & $\AA$ & 7.24 \\
\hline & $R=\frac{G_{001}}{G_{001}}$ & - & 1 \\
\hline \multirow{4}{*}{ [010] PBC } & $a_{P}$ & $\AA$ & 4.11 \\
\hline & $a_{E}$ & $\AA$ & 5.97 \\
\hline & $\alpha_{i, i+1}$ & $\circ$ & 54.0 \\
\hline & $\phi_{k}$ & $\mathrm{kT}$ & 0.75 \\
\hline \multirow{4}{*}{ [110] PBC } & $a_{P}$ & $\AA$ & 4.83 \\
\hline & $a_{E}$ & $\AA$ & 5.08 \\
\hline & $\alpha_{i, i+1}$ & $\circ$ & 72.1 \\
\hline & $\phi_{k}$ & $\mathrm{kT}$ & 1.00 \\
\hline \multirow{4}{*}{ [110] PBC } & $a_{P}$ & $\bar{\AA}$ & 4.83 \\
\hline & $a_{E}$ & $\AA$ & 5.08 \\
\hline & $\alpha_{i, i+1}$ & $\circ$ & 54.0 \\
\hline & $\phi_{k}$ & $\mathrm{kT}$ & 1.00 \\
\hline
\end{tabular}

\begin{tabular}{|c|c|c|c|}
\hline \multicolumn{4}{|c|}{ Face $(20 \overline{1})$} \\
\hline Parameter & & Units & Value \\
\hline \multirow{4}{*}{$(\sigma<0.36)$} & $h$ & $\AA$ & 4.09 \\
\hline & $R=\frac{G_{20 \overline{1}}}{G_{000}}$ & - & 1.24 \\
\hline & $\sigma_{2 D}$ & - & 0.36 \\
\hline & $\sigma_{n_{C}=10}$ & - & 0.70 \\
\hline \multirow{4}{*}{ [010] PBC } & $a_{P}$ & $\AA$ & 7.27 \\
\hline & $a_{E}$ & $\AA$ & 5.97 \\
\hline & $\alpha_{i, i+1}$ & 0 & 67.7 \\
\hline & $\phi_{k}$ & $\mathrm{kT}$ & 0.75 \\
\hline \multirow{4}{*}[\overline{1}\overline{1}\overline{2}]{$\mathrm{PBC}$} & $a_{P}$ & $\AA$ & 5.53 \\
\hline & $a_{E}$ & $\AA$ & 7.86 \\
\hline & $\alpha_{i, i+1}$ & 0 & 44.6 \\
\hline & $\phi_{k}$ & $\mathrm{kT}$ & 0.31 \\
\hline \multirow{4}{*}[\overline{1}1\overline{2}]{$\mathrm{PBC}$} & $a_{P}$ & $\AA$ & 5.53 \\
\hline & $a_{E}$ & $\AA$ & 7.86 \\
\hline & $\alpha_{i, i+1}$ & $\circ$ & 67.7 \\
\hline & $\phi_{k}$ & $\mathrm{kT}$ & 0.31 \\
\hline
\end{tabular}

Face (110)

\begin{tabular}{clcc}
\hline Parameter & & Units & Value \\
\hline & $h$ & $\AA$ & 4.50 \\
$(\sigma<0.30)$ & $R=\frac{G_{110}}{G_{001}}$ & - & 1.18 \\
$(\sigma=0.35)$ & $R=\frac{G_{110}}{G_{001}}$ & - & 1.56 \\
& $\sigma_{2 D}$ & - & 0.30 \\
& $\sigma_{n_{C}=10}$ & - & 0.67 \\
\hline & $a_{P}$ & $\AA$ & 4.55 \\
{$[001]$ PBC } & $a_{E}$ & $\AA$ & 8.67 \\
& $\alpha_{i, i+1}$ & $\circ$ & 35.3 \\
& $\phi_{k}$ & $\mathrm{kT}$ & 0.26 \\
\hline & $a_{P}$ & $\AA$ & 5.02 \\
{$[\overline{1} 1 \overline{2}] \mathrm{PBC}$} & $a_{E}$ & $\AA$ & 7.86 \\
& $\alpha_{i, i+1}$ & $\circ$ & 81.1 \\
& $\phi_{k}$ & $\mathrm{kT}$ & 0.31 \\
\hline & $a_{P}$ & $\AA$ & 7.77 \\
{$[\overline{1} 10] \mathrm{PBC}$} & $a_{E}$ & $\AA$ & 5.08 \\
& $\alpha_{i, i+1}$ & $\circ$ & 63.6 \\
& $\phi_{k}$ & $\mathrm{kT}$ & 1.00 \\
\hline
\end{tabular}

Face $(11 \overline{1})$

\begin{tabular}{clcr}
\hline Parameter & & Units & Value \\
\hline & $h$ & $\AA$ & 4.65 \\
$(\sigma<0.13)$ & $R=\frac{G_{11 \overline{1}}}{G_{001}}$ & - & 1.13 \\
$(\sigma=0.16)$ & $R=\frac{G_{11 \overline{1}}}{G_{001}}$ & - & 2.10 \\
$(\sigma=0.35)$ & $R=\frac{G_{11 \overline{1}}}{G_{001}}$ & - & 5.64 \\
& $\sigma_{2 D}$ & - & 0.13 \\
& $\sigma_{n_{C}=10}$ & - & 0.49 \\
\hline & $a_{P}$ & $\AA$ & 4.76 \\
{$[101] \mathrm{PBC}$} & $a_{E}$ & $\AA$ & 8.02 \\
& $\alpha_{i, i+1}$ & $\circ$ & 69.7 \\
& $\phi_{k}$ & $\mathrm{kT}$ & 0.37 \\
\hline & $a_{P}$ & $\AA$ & 7.52 \\
{$[\overline{1} 10] \mathrm{PBC}$} & $a_{E}$ & $\AA$ & 5.08 \\
& $\alpha_{i, i+1}$ & $\circ$ & 73.0 \\
& $\phi_{k}$ & $\mathrm{kT}$ & 1.00 \\
\hline & $a_{P}$ & $\AA$ & 4.86 \\
{$[\overline{1} \overline{1} \overline{2}] \mathrm{PBC}$} & $a_{E}$ & $\AA$ & 7.86 \\
& $\alpha_{i, i+1}$ & $\circ$ & 37.3 \\
& $\phi_{k}$ & $\mathrm{kT}$ & 0.31 \\
\hline
\end{tabular}


Table 9: Naphthalene faces, grown from cyclohexane

\begin{tabular}{|c|c|c|c|}
\hline \multicolumn{4}{|c|}{ Face (001) } \\
\hline \multirow[t]{5}{*}{ Parameter } & & Units & Value \\
\hline & $h$ & $\AA$ & 7.24 \\
\hline & $R=\frac{G_{001}}{G_{001}}$ & - & 1 \\
\hline & $\sigma_{2 D}$ & - & $\mathrm{n} / \mathrm{a}$ \\
\hline & $\sigma_{n_{C}=10}$ & - & 0.96 \\
\hline \multirow{4}{*}{ [010] PBC } & $a_{P}$ & $\AA$ & 4.11 \\
\hline & $a_{E}$ & $\AA$ & 5.97 \\
\hline & $\alpha_{i, i+1}$ & $\circ$ & 54.0 \\
\hline & $\phi_{k}$ & $\mathrm{kT}$ & 0.11 \\
\hline \multirow{4}{*}{ [110] PBC } & $a_{P}$ & $\AA$ & 4.83 \\
\hline & $a_{E}$ & $\AA$ & 5.08 \\
\hline & $\alpha_{i, i+1}$ & $\circ$ & 72.1 \\
\hline & $\phi_{k}$ & $\mathrm{kT}$ & 0.20 \\
\hline \multirow{4}{*}{ [110] PBC } & $a_{P}$ & $\AA$ & 4.83 \\
\hline & $a_{E}$ & $\AA$ & 5.08 \\
\hline & $\alpha_{i, i+1}$ & $\circ$ & 54.0 \\
\hline & $\phi_{k}$ & $\mathrm{kT}$ & 0.20 \\
\hline
\end{tabular}

\begin{tabular}{|c|c|c|c|}
\hline \multicolumn{4}{|c|}{ Face $(20 \overline{1})$} \\
\hline Parameter & & Units & Value \\
\hline \multirow{4}{*}{$(\sigma<0.011)$} & $h$ & $\AA$ & 4.09 \\
\hline & $R=\frac{G_{20 \overline{1}}}{G_{001}}$ & - & 2.32 \\
\hline & $\sigma_{2 D}$ & - & 0.011 \\
\hline & $\sigma_{n_{C}=10}$ & - & 0.13 \\
\hline \multirow{4}{*}{ [010] PBC } & $a_{P}$ & $\AA$ & 7.27 \\
\hline & $a_{E}$ & $\AA$ & 5.97 \\
\hline & $\alpha_{i, i+1}$ & $\circ$ & 67.7 \\
\hline & $\phi_{k}$ & $\mathrm{kT}$ & 0.11 \\
\hline \multirow{4}{*}[\overline{1}\overline{1}\overline{2}]{$\mathrm{PBC}$} & $a_{P}$ & $\AA$ & 5.53 \\
\hline & $a_{E}$ & $\AA$ & 7.86 \\
\hline & $\alpha_{i, i+1}$ & $\circ$ & 44.6 \\
\hline & $\phi_{k}$ & $\mathrm{kT}$ & 0.01 \\
\hline \multirow{4}{*}[\overline{1}1\overline{2}]{$\mathrm{PBC}$} & $a_{P}$ & $\AA$ & 5.53 \\
\hline & $a_{E}$ & $\AA$ & 7.86 \\
\hline & $\alpha_{i, i+1}$ & $\circ$ & 67.7 \\
\hline & $\phi_{k}$ & $\mathrm{kT}$ & 0.01 \\
\hline
\end{tabular}

\begin{tabular}{llcr}
\hline \multicolumn{4}{l}{ Face $(110)$} \\
\hline Parameter & & Units & Value \\
\hline & $h$ & $\AA$ & 4.50 \\
$(\sigma=0.0061)$ & $R=\frac{G_{110}}{G_{001}}$ & - & 2.13 \\
$(\sigma=0.0070)$ & $R=\frac{G_{110}}{G_{001}}$ & - & 3.83 \\
$(\sigma=0.0082)$ & $R=\frac{G_{110}}{G_{001}}$ & - & 18.3 \\
& $\sigma_{2 D}$ & - & 0.0055 \\
& $\sigma_{n_{C}=10}$ & - & 0.11 \\
\hline & $a_{P}$ & $\AA$ & 4.55 \\
{$[001] \mathrm{PBC}$} & $a_{E}$ & $\AA$ & 8.67 \\
& $\alpha_{i, i+1}$ & $\circ$ & 35.3 \\
& $\phi_{k}$ & $\mathrm{kT}$ & 0.03 \\
\hline & $a_{P}$ & $\AA$ & 5.02 \\
{$[\overline{1} \overline{2}] \mathrm{PBC}$} & $a_{E}$ & $\AA$ & 7.86 \\
& $\alpha_{i, i+1}$ & $\circ$ & 81.1 \\
& $\phi_{k}$ & $\mathrm{kT}$ & 0.01 \\
\hline & $a_{P}$ & $\AA$ & 7.77 \\
{$[\overline{1} 10] \mathrm{PBC}$} & $a_{E}$ & $\AA$ & 5.08 \\
& $\alpha_{i, i+1}$ & $\circ$ & 63.6 \\
& $\phi_{k}$ & $\mathrm{kT}$ & 0.20 \\
\hline
\end{tabular}




\section{References}

[1] Kuvadia, Z. B.; Doherty, M. F. Cryst. Growth Des. 2011, 11, 2780-2802.

[2] Reviakine, I.; Georgiou, D. K.; Vekilov, P. G. J. Am. Chem. Soc. 2003, 125, 11684-11693.

[3] Petsev, D. N.; Chen, K.; Gliko, O.; Vekilov, P. G. Proc. Natl. Acad. Sci. U. S. A. 2003, 100, $792-796$.

[4] Sours, R. E.; Zellelow, A. Z.; Swift, J. A. J. Phys. Chem. B 2005, 109, 9989-9995.

[5] Lovette, M. A.; Doherty, M. F. Phys. Rev. E 2012, 85, 021604.

[6] Charbonneau, G. P.; Delugeard, Y. Acta Crystallogr., Sect. B: Struct. Crystallogr. Cryst. Chem. 1977, 33, 1586-1588.

[7] Sasse, K.; N'guimbi, J.; Jose, J.; Merlin, J. Thermochim. Acta 1989, 146, 53 - 61.

[8] Jetten, L.; Human, H.; Bennema, P.; Van der Eerden, J. J. Cryst. Growth 1984, 68, 503-516.

[9] Housty, J.; Hospital, M. Acta Crystallogr. 1965, 18, 693-697.

[10] Albyn, K. C. J. Chem. Eng. Data 2001, 46, 1415-1416.

[11] Davey, R. J.; Black, S. N.; Logan, D.; Maginn, S. J.; Fairbrother, J. E.; Grant, D. J. W. J. Chem. Soc., Faraday Trans. 1992, 88, 3461-3466.

[12] Eilerman, D.; Rudman, R. Acta Crystallogr., Sect. B: Struct. Crystallogr. Cryst. Chem. 1979, $35,2458-2460$.

[13] Barone, G.; Gatta, G. D.; Ferro, D.; Piacente, V. J. Chem. Soc., Faraday Trans. 1990, 86, $75-79$.

[14] Bernardo, A.; Giulietti, M. Chem. Eng. Res. Des. 2010, 88, 1356-1364.

[15] Genbo, S.; Zhengdong, L.; Rfen, P.; Dexiang, W.; Tongqin, Y. J. Cryst. Growth 1992, 119, $368-370$.

[16] Brock, C. P.; Dunitz, J. D. Acta Crystallogr., Sect. B: Struct. Crystallogr. Cryst. Chem. 1982, $38,2218-2228$. 
[17] Murray, J.; Cavell, K.; Hill, J. Thermochim. Acta 1980, 36, 97 - 101.

[18] Grimbergen, R. F. P.; Reedijk, M. F.; Meekes, H.; Bennema, P. J. Phys. Chem. B 1998, 102, $2646-2653$. 